MATHEMATICS OF COMPUTATION

Volume 71, Number 238, Pages 743-759

S 0025-5718(01)01404-1

Article electronically published on December 21, 2001

\title{
AN ALGORITHM \\ FOR FINDING ALL PREPROJECTIVE COMPONENTS OF THE AUSLANDER-REITEN QUIVER
}

\author{
PETER DRÄXLER AND KLARA KÖGERLER
}

\begin{abstract}
The Auslander-Reiten quiver of a finite-dimensional associative algebra $A$ encodes information about the indecomposable finite-dimensional representations of $A$ and their homomorphisms. A component of the AuslanderReiten quiver is called preprojective if it does not admit oriented cycles and each of its modules can be shifted into a projective module using the AuslanderReiten translation. Preprojective components play an important role in the present research on algebras of finite and tame representation type. We present an algorithm which detects all preprojective components of a given algebra.
\end{abstract}

\section{INTRODUCTION}

It is one of the main aims of representation theory to understand the category of representations of an algebra $A$ over a commutative ring $k$. We consider the case that $k$ is an algebraically closed field and $A$ is associative and finite-dimensional as $k$-space. Moreover, we are only interested in finite-dimensional representations which we consider as finite-dimensional right $A$-modules. Together with the $A$ homomorphisms these modules form the category mod $-A$.

The key which allows us to study $\bmod -A$ with combinatorial algorithms is the observation that $A$ and to a large extend also $\bmod -A$ can be studied using directed graphs known as quivers. Namely, it was observed by Gabriel (see [Ga1], Ga2]) that any basic finite-dimensional algebra over an algebraically closed field $k$ is of the shape $k[Q] / I$ where $Q$ is a finite quiver and $I$ is an admissible ideal of the path algebra $k[Q]$. Since any finite-dimensional algebra is Morita equivalent to a basic algebra and Morita equivalent algebras have equivalent module categories, it is no loss of generality to study only the representation theory of basic algebras.

The quiver describing mod-A is usually called the Auslander-Reiten quiver of $A$. Its set of vertices is formed by picking precisely one representative from each isomorphism class of indecomposable modules, and the number of arrows from $X$ to $Y$ is given by the $k$-dimension of the space $\operatorname{rad}_{A}(X, Y) / \operatorname{rad}_{A}^{2}(X, Y)$. Note that the Auslander-Reiten quiver is even a translation quiver, meaning that it carries an additional structure, namely the Auslander-Reiten translation $\tau_{A}$.

In general, it is hard to calculate components of the Auslander-Reiten quiver. One reason is that the calculation of $\tau_{A}$ involves the transpose functor (see [ARS]),

Received by the editor April 6, 1999 and, in revised form, July 7, 2000.

2000 Mathematics Subject Classification. Primary 16G20, 16G70; Secondary 05C38, 05E99.

Key words and phrases. Representations of algebras, Auslander-Reiten quiver, preprojective components. 
for which no practicable way of implementation is known. The other reason is that the Auslander-Reiten quiver usually has many oriented cycles, and therefore no inductive construction is possible because there are no natural starting points. One exception is the so-called preprojective components, which are components without oriented cycles where every vertex can be shifted into a projective module by some power of $\tau_{A}$.

Preprojective components play a decisive role in the study of finite-dimensional algebras. The reason is that in many cases (e.g. for algebras of finite representation type, see [BGRS]) covering techniques (see [BG]) allow us to reduce the study of an algebra to the study of an algebra having a preprojective component in its Auslander-Reiten quiver. The Bautista-Larrión separation criterion ([B] ; see also Bo] provides a handy sufficient condition to ensure the existence of a preprojective component. Nevertheless, there are many algebras that do not satisfy the separation criterion whereas their Auslander-Reiten quivers do have preprojective components.

We will see in Section 3 that concerning preprojective components it suffices to consider algebras $A=k[Q] / I$ where $Q$ is cycle-free, i.e. there are no oriented cycles in $Q$. The aim of this paper is to present an inductive algorithm which produces an initial subquiver $Q^{\star}$ of $Q$ such that the algebra $A^{\star}=k\left[Q^{\star}\right] / I \cap k\left[Q^{\star}\right]$ has the following properties:

- The Auslander-Reiten quiver of $A^{\star}$ has preprojective components which contain all indecomposable projective modules over $A^{\star}$.

- A preprojective component of the Auslander-Reiten quiver of $A^{\star}$ is a preprojective component of the Auslander-Reiten quiver of $A$ if and only if it does not contain any indecomposable radical summand of an indecomposable projective module associated with a direct neighbour of $Q^{\star}$ in $Q$.

- All preprojective components of the Auslander-Reiten quiver of $A$ appear as above.

Since modules in preprojective components are characterised by their dimension vectors and by $\mathrm{KP}$ only a finite initial piece of a preprojective component can contain a given module, this is a finite algorithm which allows us to check the existence of preprojective components and to construct all of them.

Implementations of algorithms for the calculation of preprojective components (in case they exist) are contained in the CREP program system. (The CREP system and information about it can be found in the WWW under the URL http://www.mathematik.uni-bielefeld.de/birep/crep.html.) These implementations can be used to perform our inductive algorithm step by step. A completely automatic implementation of our algorithm which only needs the quiver $Q$ and the dimension vectors of the indecomposable projective modules and the indecomposable direct summands of their radicals as input data is in preparation.

The inductive calculation of preprojective components is very fast, because only comparisons and additions of vectors with short integer entries have to be performed. These vectors are of length $q$, which is the number of vertices of the quiver $Q$ where $A=k[Q] / I$ is the given algebra. Thus the running time does not depend on the number of arrows and is quadratic in $q$, since for every inductive step at most $q$ dimension vectors of length $q$ have to be calculated. Since the calculations of the new dimension vectors are independent of each other, on a parallel computer the running time will even be linear in $q$. 
Let us outline the contents of the paper. In Section 2 we provide the necessary definitions and preliminaries. For more background we refer to [ARS, GR] and Ri]. Section 3 is devoted to the study of completely preprojective algebras which are defined by the property that every indecomposable projective module appears in some preprojective component. In Section 4 we present our algorithm and show that the first of the above properties holds. Finally, in Section 5 we establish the uniqueness of $A^{\star}$ and prove the two remaining properties.

\section{Definitions AND PRELIMINARIES}

2.1. Let $A$ be a basic finite-dimensional $k$-algebra with a chosen decomposition $1=\sum_{i=1}^{n} e_{i}$ of the unit element of $A$ into orthogonal primitive idempotents. We form a $k$-linear category $\mathcal{A}$ using $\{1, \ldots, n\}$ as set of objects and $e_{y} A e_{x}$ as space $\mathcal{A}(x, y)$ of morphisms from $x$ to $y$. The composition is the multiplication in $A$. The category $\bmod -A$ of finite-dimensional right $A$-modules is equivalent to the category $\bmod -\mathcal{A}$ of $k$-linear contravariant functors from $\mathcal{A}$ to the category $\bmod -k$ of finite-dimensional $k$-spaces. We will denote the functors in this category as $\mathcal{A}$ modules. The equivalence sends an $A$-module $M$ to the functor which associates the space $M e_{x}$ to the object $x$ and the right multiplication by $a$ with each morphism $a \in e_{y} A e_{x}$.

Note that $\mathcal{A}$ is a spectroid in the sense of [GR], meaning that $\mathcal{A}$ is a $k$-linear category with finite-dimensional morphism spaces such that the endomorphism algebra of each object is local and any two objects are isomorphic if and only if they are equal. The assumption that the algebra $A$ we started with is finite-dimensional is reflected by the fact that the spectroid $\mathcal{A}$ is finite, i.e. has a finite set of objects. We will follow $\mathrm{GR}$ by considering finite spectroids $\mathcal{A}$ rather than finite-dimensional algebras $A$.

If we choose a minimal complete set of representatives of isomorphism classes of indecomposable modules in $\bmod -\mathcal{A}$, then the induced full subcategory ind $-\mathcal{A}$ will also be a spectroid, but usually with an infinite set of objects.

Examples for objects in ind $-\mathcal{A}$ are the indecomposable projective modules $P_{x}=$ $\mathcal{A}(-, x)$ and the indecomposable injective objects $I_{x}=\mathrm{D} \mathcal{A}(x,-)$ for all objects $x$ of $\mathcal{A}$, where $\mathrm{D}=\operatorname{Hom}_{k}(-, k)$ is the usual duality.

2.2. If $\mathcal{S}$ is an arbitrary spectroid, then its Jacobson radical $\operatorname{rad} \mathcal{S}$ consists of all nonisomorphisms in $\mathcal{S}$. The quiver $Q_{\mathcal{S}}$ of $\mathcal{S}$ has the set of objects of $\mathcal{S}$ as set of vertices, and the number of arrows from $x$ to $y$ is the dimension of the space $\operatorname{rad} \mathcal{S}(x, y) / \operatorname{rad}^{2} \mathcal{S}(x, y)$.

The $k$-linear path category $k Q$ of an arbitrary quiver $Q$ has the vertex set of $Q$ as objects and the set of oriented paths from $x$ to $y$ in $Q$ as basis of the morphism space $k Q(x, y)$. A $k$-linear functor $\pi: k Q_{\mathcal{S}} \rightarrow \mathcal{S}$ which is the identity on the objects and maps the set of arrows from $x$ to $y$ to a set of morphisms inducing a basis of the space $\operatorname{rad} \mathcal{S}(x, y) / \operatorname{rad}^{2} \mathcal{S}(x, y)$ is called a presentation of $\mathcal{S}$. If $\mathcal{A}$ is a finite spectroid, then any presentation $\pi: k Q_{\mathcal{A}} \rightarrow \mathcal{A}$ is full and therefore $\mathcal{A}$ can be identified with $k Q_{\mathcal{A}} / I$, where $I$ is the kernel of $\pi$.

2.3. Due to results of Auslander and Reiten the quiver of the spectroid ind $-\mathcal{A}$ for a finite spectroid $\mathcal{A}$ carries the additional structure of a translation, i.e. it is a translation quiver, which is usually called the Auslander-Reiten quiver and denoted by $\Gamma_{\mathcal{A}}$. In order to introduce the translation, let us first recall the notion of almost 
split morphisms and minimal morphisms in $\bmod -\mathcal{A}$. A morphism $g: Y \rightarrow Z$ in $\bmod -\mathcal{A}$ is said to be right almost split if it is not a retraction but each morphism $g^{\prime}: Y^{\prime} \rightarrow Z$ which is not a retraction factors as $g^{\prime}=g h$. It is easy to see that the existence of such a $g$ forces $Z$ to be indecomposable. A morphism $g: Y \rightarrow Z$ is called right minimal if each endomorphism $h: Y \rightarrow Y$ satisfying $g=g h$ has to be an isomorphism. Left almost split and left minimal morphisms are defined in the dual fashion.

The importance of right minimal and right almost split morphisms (and dually the left minimal and left almost split morphisms) comes from the following fact. If $Z$ and $U$ are objects in ind $-\mathcal{A}$ and $g: Y \rightarrow Z$ is a right minimal and right almost split morphism, then the number of arrows from $U \rightarrow Z$ in $\Gamma_{\mathcal{A}}$ equals the multiplicity of $U$ as direct summand of $Y$.

For each object $x$ of $\mathcal{A}$ the inclusion $\iota_{x}$ of the submodule $\operatorname{rad} P_{x}=P_{x} \operatorname{rad} \mathcal{A}$ into $P_{x}$ is right minimal and right almost split. Dually, the projection from $I_{x}$ onto the factor of $I_{x}$ by the socle of $I_{x}$ is left minimal and left almost split. If $Z$ in ind $-\mathcal{A}$ is not projective or $X$ in ind $-\mathcal{A}$ is not injective, then the fundamental result of Auslander and Reiten (see [AR]) says that there is a short exact sequence $0 \rightarrow X \stackrel{f}{\longrightarrow} Y \stackrel{g}{\longrightarrow} Z \rightarrow 0$ in $\bmod -\mathcal{A}$ (called Auslander-Reiten sequence) where $f$ is left minimal and left almost split and $g$ is right minimal and right almost split. Moreover, the modules $X$ and $Z$ uniquely determine each other. Therefore, by putting $X=\tau_{\mathcal{A}} Z$ and $Z=\tau_{\mathcal{A}}^{-} X$ we obtain mutually inverse bijections between the nonprojective objects of ind $-\mathcal{A}$ and the noninjective objects of ind $-\mathcal{A}$. The map $\tau_{\mathcal{A}}$ is called the Auslander-Reiten translation. It follows that for $Z$ and $U$ in ind- $\mathcal{A}$ with $Z$ nonprojective the number of arrows in $\Gamma_{\mathcal{A}}$ from $U$ to $Z$ equals the number of arrows from $\tau_{\mathcal{A}} Z$ to $U$. This is the defining property of a general translation quiver.

In order to avoid case by case inspections, for the indecomposable projective modules $P_{x}$ we put $\tau_{\mathcal{A}} P_{x}=0$ and for the indecomposable injective modules $I_{x}$ we put $\tau_{\mathcal{A}}^{-} I_{x}=0$.

2.4. The components of $\Gamma_{\mathcal{A}}$ are usually hard to calculate. The situation is much easier for preprojective components. A (connected) component $\Theta$ of $\Gamma_{\mathcal{A}}$ is said to be preprojective if it does not contain oriented cycles and for every vertex $Z$ of $\Theta$ there is a nonnegative integer $n$ such that $\tau_{\mathcal{A}}^{n} Z$ is projective.

For a module $M$ in $\bmod -\mathcal{A}$ the vector $\operatorname{dim} M=\left(\operatorname{dim}_{k} M(x)\right)_{x \in \mathcal{A}} \in \mathbb{Z}^{n}$ is called the dimension vector of $M$. Since for every exact sequence $0 \rightarrow X \longrightarrow Y \longrightarrow Z \rightarrow 0$ the equation $\operatorname{dim} X+\operatorname{dim} Z=\operatorname{dim} Y$ holds, the dimension vector of the end term $Z$ of an Auslander-Reiten sequence can be easily calculated once the dimension vectors of the start term $X$ and the indecomposable summands of the middle term $Y$ are known.

By writing $\mathcal{A}$ as $k Q_{\mathcal{A}} / I$ the modules $P_{x}=\mathcal{A}(-, x)$ are actually known because the paths in $Q_{\mathcal{A}}$ ending in $x$ and not belonging to $\mathcal{I}$ form a finite set of generators for $P_{x}$ over $k$ which can be reduced to a basis. With some more effort one can now decompose the radicals $\operatorname{rad} P_{x}$ into indecomposable summands. Because by [Ha] an indecomposable module in a preprojective component is uniquely determined by its dimension vector, preprojective components can be calculated inductively using the dimension vectors of the indecomposable projective modules and their indecomposable radical summands. Thus these dimension vectors together with the quiver $Q_{\mathcal{A}}$ will serve as input data for our algorithm. The crucial point is 
that one has to know which indecomposable projective modules actually appear in preprojective components. This is the question we will answer.

2.5. We need some device which allows us to induce Auslander-Reiten sequences from subspectroids. Let $\mathcal{B}$ be a full subspectroid of the finite spectroid $\mathcal{A}$; then the canonical restriction functor $\operatorname{res}_{\mathcal{B}}: \bmod -\mathcal{A} \rightarrow \bmod -\mathcal{B}$ has a right adjoint $R$ and a left adjoint $L$ which both are functors $\bmod -\mathcal{B} \rightarrow \bmod -\mathcal{A}$. For an object $x$ of $\mathcal{A}$ and a module $U$ in $\bmod -\mathcal{B}$ we have $R U(x)=\operatorname{Hom}_{\mathcal{B}}\left(\operatorname{res}_{\mathcal{B}} P_{x}, U\right)$ and $L U(x)=U \otimes_{\mathcal{B}}$ $\operatorname{res}_{\mathcal{B}} \mathcal{A}(x,-) \cong \operatorname{DHom}_{\mathcal{B}}\left(U, \operatorname{res}_{\mathcal{B}} I_{x}\right)$. Consequently, the functor $R$ is left exact and maps the indecomposable injective module $I_{x}$ over $\mathcal{B}$ to the corresponding module $I_{x}$ over $\mathcal{A}$. Similarly, $L$ is right exact and maps $P_{x}$ over $\mathcal{B}$ to $P_{x}$ over $\mathcal{A}$. In $[\mathrm{Sc}]$ it is shown that for $Z$ in ind $-\mathcal{B}$ the module $L Z$ in ind $-\mathcal{A}$ satisfies $R \tau_{\mathcal{B}} Z=\tau_{\mathcal{A}} L Z$. Dually, for $X$ in ind $-\mathcal{B}$ the module $R X$ is in ind $-\mathcal{A}$ and $L \tau_{\mathcal{B}}^{-} X=\tau_{\mathcal{A}}^{-} R X$.

For a spectroid $\mathcal{S}$ a full subspectroid $\mathcal{T}$ is called initial provided that, for any nonzero map $f: x \rightarrow y$ in $\mathcal{S}$, if $y$ lies in $\mathcal{T}$, then also $x$ lies in $\mathcal{T}$. In an analogous way a full subquiver $Q^{\prime}$ of a quiver $Q$ is said to be initial if, for any arrow $x \rightarrow y$, if $y$ lies in $Q^{\prime}$, then also $x$ lies in $Q^{\prime}$.

Let $\mathcal{B}$ be a full subspectroid of a finite spectroid $\mathcal{A}$ with $\mathcal{A}=k Q_{\mathcal{A}} / I$. Then $\mathcal{B}$ is initial if and only if the full subquiver $Q^{\prime}$ of $Q_{\mathcal{A}}$ associated with the objects of $\mathcal{B}$ is initial. In this case $Q_{\mathcal{B}}=Q^{\prime}$ and $\mathcal{B}=k Q^{\prime} / I \cap k Q^{\prime}$.

For an initial subspectroid $\mathcal{B}$ of $\mathcal{A}$ every module $N$ over $\mathcal{B}$ can be considered as a module over $\mathcal{A}$ by putting $N(x)=0$ for all $x$ which belong to $\mathcal{A}$ but not to $\mathcal{B}$. Moreover, this inclusion $\bmod -\mathcal{B} \rightarrow \bmod -\mathcal{A}$ coincides with the functor $L$. Therefore the formulas from [Sc] degenerate to $R \tau_{\mathcal{B}} Z=\tau_{\mathcal{A}} Z$ and $\tau_{\mathcal{B}}^{-} X=\tau_{\mathcal{A}}^{-} R X$. In contrast to $L N$, the module $R N$ is usually not isomorphic to $N$. Nevertheless, because $R N(x)=N(x)$ for all $x$ in $\mathcal{B}$, there is a canonical inclusion of $N$ into $R N$, which allows us to consider $N$ as submodule of $R N$.

We will frequently use the fact that an Auslander-Reiten sequence $0 \rightarrow X \longrightarrow$ $Y \longrightarrow Z \rightarrow 0$ in $\bmod -\mathcal{A}$ where $X$ and $Z$ belong to $\bmod -\mathcal{B}$ is actually also an Auslander-Reiten sequence in $\bmod -\mathcal{B}$.

2.6. For a preprojective component $\Theta$ of $\Gamma_{\mathcal{A}}$ we denote by $\mathcal{S}(\Theta)$ the full subspectroid of ind $-\mathcal{A}$ associated with the vertices of $\Theta$. The defining property of right minimal and right almost split maps shows that any presentation $k \Theta \rightarrow \mathcal{S}(\Theta)$ is full. With the same argument one obtains that $\mathcal{S}(\Theta)$ is an initial subspectroid of ind $-\mathcal{A}$.

\section{Completely preprojective algebras}

3.1. A finite-dimensional algebra or equivalently a finite spectroid $\mathcal{A}$ is called completely preprojective if every indecomposable projective module lies in a preprojective component. Note that $\mathcal{A}$ (or equivalently $Q_{\mathcal{A}}$ ) is not assumed to be connected. This is quite uncommon in the representation theory of algebras, but we will see that this is essential for our purposes because we will have to build up quivers inductively from smaller subquivers which usually will not be connected even if the final quiver is. A preprojective component $\Theta$ of $\Gamma_{\mathcal{A}}$ is said to be complete if all indecomposable projective modules $P_{x}$ belong to $\Theta$.

If $\mathcal{A}$ is completely preprojective, then any cycle in $Q_{\mathcal{A}}$ would give rise to a cycle in a preprojective component of $\Gamma_{\mathcal{A}}$. Hence the quiver $Q_{\mathcal{A}}$ of a completely preprojective finite spectroid $\mathcal{A}$ has to be cycle-free, i.e. it does not admit oriented 
cycles. Similarly, one sees that $Q_{\mathcal{A}}$ is connected if and only if there is precisely one preprojective component, which is obviously complete.

Proposition 1. Let $\mathcal{A}$ be a finite spectroid and $\Theta$ a preprojective component of the Auslander-Reiten quiver $\Gamma_{\mathcal{A}}$. If we denote by $\mathcal{B}$ the full subcategory of $\mathcal{A}$ associated with all objects $x$ such that $Z(x) \neq 0$ for some $Z$ in $\Theta$, then the following assertions hold:

(1) $\mathcal{B}=\left\{x \in \mathcal{A} \mid P_{x} \in \Theta\right\}$.

(2) $\mathcal{B}$ is an initial subspectroid of $\mathcal{A}$.

(3) $\tau_{\mathcal{B}}^{-} X=\tau_{\mathcal{A}}^{-} X$ and $\tau_{\mathcal{B}} X=\tau_{\mathcal{A}} X$ for each module $X$ in $\Theta$.

(4) $\Theta$ is a complete preprojective component of $\Gamma_{\mathcal{B}}$.

(5) For each $y$ in $\mathcal{A}$ and not in $\mathcal{B}$ none of the indecomposable direct summands of $\operatorname{res}_{\mathcal{B}} P_{y}$ lies in $\Theta$.

Proof. (1) If $P_{x}$ is in $\Theta$, then $P_{x}(x)=\mathcal{A}(x, x) \neq 0$ and therefore $x$ is in $\mathcal{B}$. Conversely, if $Z(x) \neq 0$ for $Z$ in $\Theta$, then by the Yoneda lemma $\operatorname{Hom}_{\mathcal{A}}\left(P_{x}, Z\right) \neq 0$ holds. Since $\mathcal{S}(\Theta)$ is initial, also $P_{x}$ belongs to $\Theta$.

(2) Suppose $\mathcal{A}(x, y) \neq 0$ and $P_{y}$ in $\Theta$. Again by the Yoneda lemma $\operatorname{Hom}_{\mathcal{A}}\left(P_{x}, P_{y}\right)$ $\neq 0$, and therefore $P_{x}$ is in $\Theta$.

(3) For $Z$ in $\Theta$ we first consider the case that $Z$ is not projective, and we dispose of an Auslander-Reiten sequence $0 \rightarrow X \longrightarrow Y \longrightarrow Z \rightarrow 0$ in $\bmod -\mathcal{A}$. On the other hand, $Z$ is also a $\mathcal{B}$-module and there is an Auslander-Reiten sequence $0 \rightarrow X^{\prime} \longrightarrow Y^{\prime} \longrightarrow Z \rightarrow 0$ in mod-B. Because for each indecomposable summand $U$ of $Y$ there is a nonzero morphism from $U$ to $Z$ and from $X$ to $U$, also $X$ and $Y$ are $\mathcal{B}$-modules, and therefore $\tau_{\mathcal{A}} Z=X=X^{\prime}=\tau_{\mathcal{B}} Z$. For $Z$ projective we get $R \tau_{\mathcal{B}} X=\tau_{\mathcal{A}} Z=0$, and therefore also $\tau_{\mathcal{B}} X=0$.

If $X$ is a noninjective module in $\Theta$, then the same arguments as above yield $\tau_{\mathcal{B}}^{-} X=\tau_{\mathcal{A}}^{-} X$. In case $X$ is injective, then $\tau_{\mathcal{A}}^{-} X=0$. But the module $R X$ is injective as well, and thus $\tau_{\mathcal{B}}^{-} X=\tau_{\mathcal{A}}^{-} R X=0$.

(4) follows from (1) and (3) immediately.

(5) Let $y$ be an object of $\mathcal{A}$ but not of $\mathcal{B}$. We assume that there is an indecomposable direct summand $U$ of $\operatorname{res}_{\mathcal{B}} P_{y}$ which lies in $\Theta$, and obtain $0 \neq \operatorname{Hom}_{\mathcal{B}}\left(\operatorname{res}_{\mathcal{B}} P_{y}, U\right)$ $=R U(y)$. Hence the canonical inclusion of $U$ into the indecomposable module $R U$ is not an isomorphism, and thus $U$ cannot be an injective $\mathcal{A}$-module. By (3) the nonzero module $X=\tau_{\mathcal{A}}^{-} U$ lies in $\Theta$. The equation $U=\tau_{\mathcal{A}} X=R \tau_{\mathcal{B}} X=R U$ gives a contradiction.

3.2. Proposition 1 shows that preprojective components can always be found as complete preprojective components for some initial subspectroid. After a preparatory lemma we will use this result to show that we only need to consider finite spectroids whose quivers are cycle-free.

Lemma 2. Let $\mathcal{B}$ be an initial subspectroid of a finite spectroid $\mathcal{A}$. Suppose that $\Theta$ is a preprojective component of $\Gamma_{\mathcal{B}}$. If for all $y$ in $\mathcal{A}$ but not in $\mathcal{B}$ the indecomposable direct summands $U$ of $\operatorname{res}_{\mathcal{B}} P_{y}$ do not belong to $\Theta$, then $\Theta$ is a preprojective component of $\Gamma_{\mathcal{A}}$.

Proof. Since $\mathcal{S}(\Theta)$ is initial in ind $-\mathcal{B}$, the assumption on the summands $U$ of the restrictions $\operatorname{res}_{\mathcal{B}} P_{y}$ shows that $R X=X$ for all $X$ in $\Theta$, and hence $\tau_{\mathcal{A}} X=\tau_{\mathcal{B}} X$, $\tau_{\mathcal{A}}^{-} X=\tau_{\mathcal{B}}^{-} X$. This implies that the Auslander-Reiten sequences constituting $\Theta$ remain Auslander-Reiten sequences over $\mathcal{A}$. Thus it remains to show that $\Theta$ is 
closed under neighbours in $\Gamma_{\mathcal{A}}$. For this reason we prove that for an arrow $X \rightarrow Y$ in $\Gamma_{\mathcal{A}}$ the start term $X$ lies in $\Theta$ if and only if the end term $Y$ lies in $\Theta$. We only establish one direction, because the proof of the other is similar. Suppose that $X$ belongs to $\Theta$, and consider a left minimal and left almost split map $X \rightarrow M$. It suffices to show that $M$ is a $\mathcal{B}$-module. In case $X$ is not injective, then as seen above $\tau_{\mathcal{A}}^{-} X$ and consequently $M$ are $\mathcal{B}$-modules. For $X$ injective we know that $M=X / \operatorname{soc} X$ is a $\mathcal{B}$-module as well.

For an arbitrary finite spectroid $\mathcal{A}$ we denote by $\mathcal{A}^{\text {init }}$ the full subspectroid given by all objects $x$ of $\mathcal{A}$ such that no predecessor of $x$ in $Q_{\mathcal{A}}$ lies on an oriented cycle of $Q_{\mathcal{A}}$. Clearly $\mathcal{A}^{\text {init }}$ is an initial subspectroid of $\mathcal{A}$ whose quiver is cycle-free.

Theorem 3. Let $\mathcal{A}$ be a finite spectroid.

(a) If $\Gamma_{\mathcal{A}}$ has a preprojective component $\Theta$, then all modules in $\Theta$ are $\mathcal{A}^{\mathrm{init}}$ modules and $\Theta$ is a preprojective component of $\Gamma_{\mathcal{A}^{\text {init }}}$ which does not contain any indecomposable summand of a restriction $\operatorname{res}_{\mathcal{A}^{\text {init }}} P_{y}$ for all $y$ in $\mathcal{A}$ but not in $\mathcal{A}^{\text {init }}$.

(b) If $\Theta$ is a preprojective component of $\Gamma_{\mathcal{A}^{\text {init }}}$ which does not contain any indecomposable summand of a restriction res $_{\mathcal{A}^{\text {init }}} P_{y}$ for all $y$ in $\mathcal{A}$ but not in $\mathcal{A}^{\text {init }}$, then $\Theta$ is a preprojective component of $\Gamma_{\mathcal{A}}$.

Proof. The first part follows from proposition 1 because every initial completely preprojective subspectroid of $\mathcal{A}$ has to be contained in $\mathcal{A}^{\text {init }}$. The second part follows from Lemma 2

Thus when looking for preprojective components one only has to consider the initial subspectroid $\mathcal{A}^{\text {init }}$ whose quiver is cycle-free. For computational purposes it is worth noting that, for checking if the indecomposable summands of the restrictions $\operatorname{res}_{\mathcal{A}^{\text {init }}} P_{y}$ appear in preprojective components of $\Gamma_{\mathcal{A}^{\text {init }}}$, one only has to know the dimension vectors of these modules.

3.3. The next lemma will allow us to recognise complete preprojective components from finite initial pieces. We recall that in a quiver $Q$ a vertex $x$ is said to be a predecessor of a vertex $y$ if there is a path $x=x_{0} \rightarrow x_{1} \rightarrow \cdots \rightarrow x_{n}=y$ in $Q$. Moreover, $X$ is called direct predecessor of $y$ if there is an arrow $x \rightarrow y$ in $Q$. The notions of successor and direct successor are defined in the dual way.

Note that in a preprojective component $\Theta$ of $\Gamma_{\mathcal{A}}$ each module $X$ has only finitely many predecessors.

Lemma 4. Suppose that $\mathcal{A}$ is a finite spectroid with cycle-free quiver $Q_{\mathcal{A}}$ and $\Theta^{\prime}$ is a full cycle-free subquiver of $\Gamma_{\mathcal{A}}$.

If $\Theta^{\prime}$ is closed under predecessors in $\Gamma_{\mathcal{A}}$, contains all indecomposable projective $\mathcal{A}$-modules, and each vertex in $\Theta^{\prime}$ has only finitely many predecessors, then the full subquiver $\Theta$ of $\Gamma_{\mathcal{A}}$ whose vertex set consists of all nonzero modules of the shape $\tau_{\mathcal{A}}^{-n} X$ with $X$ in $\Theta^{\prime}$ and an integer $n \geq 0$ is a union of preprojective components of $\Gamma_{\mathcal{A}}$ which contains all indecomposable projectives.

In particular, if $\Theta^{\prime}$ is connected, then $\Theta$ is a complete preprojective component.

Proof. Since clearly all indecomposable projectives belong to $\Theta$, the following remain to be shown.

(1) $\Theta$ is a union of components of $\Gamma_{\mathcal{A}}$.

(2) $\Theta$ is a cycle-free quiver. 
(3) For each vertex $Z$ of $\Theta$ there exist a nonnegative integer $n$ and $x$ in $\mathcal{A}$ such that $\tau_{\mathcal{A}}^{n} Z=P_{x}$.

(1) Let $X \rightarrow Y$ be an arrow in $\Gamma_{\mathcal{A}}$. We show that $X$ belongs to $\Theta$ if and only if $Y$ belongs to $\Theta$. If $Y$ is in $\Theta$, then there is a minimal nonnegative integer $n$ with $\tau_{\mathcal{A}}^{n} Y$ in $\Theta^{\prime}$. In case there exists a nonnegative integer $m<n$ such that $\tau_{\mathcal{A}}^{m} X$ is projective, then $X$ is in $\Theta$ by definition. Otherwise we obtain an arrow $\tau_{\mathcal{A}}^{n} X \rightarrow \tau_{\mathcal{A}}^{n} Y$. Because $\Theta^{\prime}$ is closed under predecessors, $\tau_{\mathcal{A}}^{n} X$ has to lie in $\Theta^{\prime}$ and consequently $X$ in $\Theta$.

If $X$ is in $\Theta$, then we assume that $Y$ does not belong to $\Theta$, which implies that $Y$ is not projective and we obtain an Auslander-Reiten sequence $0 \rightarrow U \rightarrow V \rightarrow$ $Y \rightarrow 0$. By the definition of $\Theta$ the module $U=\tau_{\mathcal{A}} Y$ cannot belong to $\Theta$ as well. On the other hand, $X$ is a direct summand of $V$, which, as seen above, shows that its predecessor $U$ is in $\Theta$. Thus we have found a contradiction.

(2) We assume that there is a cycle $X=Z_{0} \rightarrow Z_{1} \rightarrow \cdots \rightarrow Z_{t}=X$ in $\Theta$. There is a nonnegative integer $n$ such that all $\tau_{\mathcal{A}}^{n} Z_{j}$ are nonzero and at least one of these modules lies in $\Theta^{\prime}$. Because $\Theta^{\prime}$ is closed under predecessors, then the whole cycle lies in $\Theta^{\prime}$, which is a contradiction.

(3) By definition, for $X$ in $\Theta$ there exists $n$ with $\tau_{\mathcal{A}}^{n} X$ in $\Theta^{\prime}$. Because each point of the cycle-free quiver $\Theta^{\prime}$ has only finitely many predecessors, we find another $m$ such that $\tau_{\mathcal{A}}^{m+n} X$ is projective.

3.4. Loosely speaking, our strategy is to build up a completely preprojective finite spectroid $\mathcal{A}$ inductively by adding vertices starting from the sources. To formulate the induction step we define $\mathcal{A}$ to be a one-point extension of the subspectroid $\mathcal{B}$ by the object $x$ if and only if $x$ is a $\operatorname{sink}$ of $Q_{\mathcal{A}}$ and the only object of $\mathcal{B}$ which does not belong to $\mathcal{A}$. This implies that $\mathcal{B}$ is initial and $\operatorname{res}_{\mathcal{B}} P_{x}=\operatorname{rad} P_{x}$.

We will need the notion of directing modules. If $\mathcal{A}$ is a finite spectroid and $X, Y$ are objects in ind $-\mathcal{A}$, then a path of length $n$ from $X$ to $Y$ in ind $-\mathcal{A}$ is a sequence $X=X_{0} \rightarrow X_{1} \rightarrow \cdots \rightarrow X_{n}=Y$ of nonzero radical morphisms $X_{i-1} \rightarrow X_{i}$ in ind $-\mathcal{A}$. The indecomposable module $X$ is called directing if there does not exist a path of length $n>0$ which starts and ends in $X$. A possibly decomposable module $Z=\bigoplus_{i=1}^{m} Z_{i}$ with $Z_{i}$ indecomposable is said to be directing if there do not exist summands $Z_{s}, Z_{t}$ and a nonprojective $Y$ in ind $-\mathcal{A}$ such that there is a path from $Z_{s}$ to $\tau_{\mathcal{A}} Y$ and a path from $Y$ to $Z_{t}$ in ind- $\mathcal{A}$. It was shown in [HR] that both concepts of directing modules coincide for indecomposables. Moreover, it was proved that for a one-point extension $\mathcal{A}$ of $\mathcal{B}$ by $x$ the projective module $P_{x}$ is directing in ind $-\mathcal{A}$ if and only if the radical $\operatorname{rad} P_{x}$ is directing in $\bmod -\mathcal{B}$.

Note that it is easy to check whether a module whose indecomposable summands are all preprojective is directing, because in this case all the involved paths in ind $-\mathcal{A}$ come from paths in preprojective components. In particular, each indecomposable module in a preprojective component is itself directing.

Recall, furthermore, that a path $Z_{0} \rightarrow Z_{1} \rightarrow \cdots \rightarrow Z_{m}$ in the Auslander-Reiten quiver $\Gamma_{\mathcal{A}}$ is said to be sectional provided $Z_{i-2} \neq \tau_{\mathcal{A}} Z_{i}$ for all $i=2, \ldots, n$.

If $\mathcal{A}$ is a completely preprojective finite spectroid, then $x$ in $\mathcal{A}$ is called a strong sink if $P_{x}$ does not have a proper projective successor in the Auslander-Reiten quiver of $\mathcal{A}$. Every strong sink of $\mathcal{A}$ is obviously a sink of $Q_{\mathcal{A}}$, but the converse does not hold in general.

Theorem 5. Let the finite spectroid $\mathcal{A}$ be a one-point extension of $\mathcal{B}$ by $x$. We decompose $\operatorname{rad} P_{x}=\bigoplus_{i=1}^{n} U_{i}$ into indecomposable summands. Then $\mathcal{A}$ is completely 
preprojective and $x$ is a strong sink of $\mathcal{A}$ if and only if $\mathcal{B}$ is completely preprojective and $\operatorname{rad} P_{x}$ has the following properties:

(a) All summands $U_{i}$ lie in preprojective components of $\Gamma_{\mathcal{B}}$.

(b) The module $\operatorname{rad} P_{x}$ is a directing $\mathcal{B}$-module.

(c) For each object $y$ of $\mathcal{B}$ and summand $U_{j}$, any path $U_{j}=Z_{0} \rightarrow Z_{1} \rightarrow \cdots \rightarrow$ $Z_{m}=P_{y}$ in $\Gamma_{\mathcal{B}}$ has to be sectional.

Proof. Let us start the proof by assuming that $\mathcal{B}$ is completely preprojective and $\operatorname{rad} P_{x}$ satisfies the conditions (a),(b),(c). We define $\Theta^{\prime \prime}$ as the full subquiver of the union of the preprojective components of $\Gamma_{\mathcal{B}}$ given by all modules $Z$ such that any path from some $U_{j}$ to $Z$ is sectional. In order to construct the quiver $\Theta^{\prime}$, we add the vertex $P_{x}$ and as many arrows from each $U_{j}$ contained in $\Theta^{\prime \prime}$ to $P_{x}$ as this module appears as summand in $\operatorname{rad} P_{x}$. We wish to apply the preceeding Lemma 4 and have to check the required properties.

By construction $\Theta^{\prime}$ is cycle-free and each vertex has only finitely many predecessors. Since $\mathcal{B}$ is completely preprojective, condition (c) shows that all modules $P_{y}$ for $y$ in $\mathcal{A}$ belong to $\Theta^{\prime}$. Thus it remains to prove that $\Theta^{\prime}$ is a full and predecessor closed subquiver of $\Gamma_{\mathcal{A}}$. Both assertions can be derived from the following claim: If $Z$ is a module in $\Theta^{\prime}$ and $g: N \rightarrow Z$ is right minimal and right almost split in $\bmod -\mathcal{A}$, then all indecomposable direct summands of $N$ belong to $\Theta^{\prime}$.

If $Z$ is not projective, then $Z$ is a $\mathcal{B}$-module in $\Theta^{\prime \prime}$ and there is an AuslanderReiten sequence $0 \rightarrow X \longrightarrow N \stackrel{g}{\longrightarrow} Z \rightarrow 0$ in $\bmod -\mathcal{A}$ but also an Auslander-Reiten sequence $0 \rightarrow X^{\prime} \longrightarrow N^{\prime} \stackrel{g}{\longrightarrow} Z \rightarrow 0$ in $\bmod -\mathcal{B}$. Assuming $X \neq X^{\prime}$, from $X=$ $\tau_{\mathcal{A}} Z=R \tau_{\mathcal{B}} Z=R X^{\prime}$ we obtain $R X^{\prime}(x)=X(x) \neq 0$. Thus $\operatorname{Hom}_{\mathcal{B}}\left(U_{j}, X^{\prime}\right) \neq 0$ for some $U_{j}$, and hence there is a path from $U_{j}$ to $X^{\prime}$ yielding a nonsectional path from $U_{j}$ to $Z$. This is a contradiction to $Z$ in $\Theta^{\prime \prime}$. Therefore all summands of $N$ belong to $\Theta^{\prime \prime}$.

In case $Z=P_{y}$ for $y$ in $\mathcal{B}$, clearly $N=\operatorname{rad} P_{y}$ lies in $\bmod -\mathcal{B}$, and the same arguments as above apply. For $Z=P_{x}$, by (a) and (b) all indecomposable summands $U_{j}$ of $\operatorname{rad} P_{z}$ lie in $\Theta^{\prime \prime}$.

In particular, we have seen that $\Theta^{\prime \prime}$ is a full and predecessor closed subquiver of $\Gamma_{\mathcal{S}}$, which finally implies that $P_{x}$ cannot have a proper projective successor.

For proving the converse we assume that $\mathcal{A}$ is completely preprojective and $x$ is a strong sink. In order to show that $\mathcal{B}$ is completely preprojective, we want to use Lemma 4 again and consider the full subquiver $\Theta^{\prime}$ of $\Gamma_{\mathcal{A}}$ whose vertex set consists of all $Z \neq P_{x}$ such that there does not exist any nonsectional path from some $U_{j}$ to $Z$. All $Z$ in $\Theta^{\prime}$ are $\mathcal{B}$-modules, because otherwise $0 \neq Z(x) \cong \operatorname{Hom}_{\mathcal{A}}\left(P_{x}, Z\right)$, and therefore there is a path from $P_{x}$ to $Z$ inducing an nonsectional path from some $U_{j}$ to $Z$. It follows that $\Theta^{\prime}$ is a cycle-free and predecessor closed full subquiver of $\Gamma_{\mathcal{B}}$ such that each vertex has only finitely many predecessors. It remains to prove that $\Theta^{\prime}$ contains all indecomposable projective $\mathcal{B}$-modules.

Let us assume the existence of some $P_{y}$ with $P_{y}$ not in $\Theta^{\prime}$. This means that there is a nonsectional path $U_{j}=Z_{0} \rightarrow Z_{1} \rightarrow \cdots \rightarrow Z_{m}=P_{y}$. All $Z_{i}$ have to be $\mathcal{B}$-modules, because otherwise $\operatorname{Hom}_{\mathcal{A}}\left(P_{x}, Z_{i}\right) \neq 0$ and the Yoneda lemma would yield that $x$ is not a strong sink. Let us choose $t$ as the smallest index such that $Z_{t}=\tau_{\mathcal{A}} Z_{t+2}$. Without loss of generality we may assume that there is no nonsectional path from $U_{j}$ to $Z_{t}$. By [HR, proposition 2] we obtain $\operatorname{Hom}_{\mathcal{B}}\left(U_{j}, Z_{t}\right) \neq$ 0 , implying $R Z_{t}(x) \neq 0$ and hence $R Z_{t} \neq Z_{t}$. On the other hand, we know that $Z_{t}=\tau_{\mathcal{A}} Z_{t+2} \cong R \tau_{\mathcal{B}} Z_{t+2}=R Z_{t}$. 
Since the preprojective module $P_{x}$ is a directing $\mathcal{A}$-module, by [HR] $\operatorname{rad} P_{x}$ is a directing $\mathcal{B}$-module, which implies (a) and (b). Finally, we have seen above that all indecomposable projective $\mathcal{B}$-modules belong to $\Theta^{\prime}$, yielding (c).

\section{The ALGORITHM}

4.1. In view of the reduction to $\mathcal{A}^{\text {init }}$ introduced in the last section, from now on we will only consider spectroids $\mathcal{A}$ whose quivers are cycle-free. We know that any preprojective component comes from a completely preprojective initial subspectroid which we could find by inductive one-point extension using Theorem 5 The problem is that for each consecutive one-point extension we need a strong sink, and it is not clear how to arrange this. Before we formulate the algorithm solving this problem, we will illustrate the difficulty by an easy example.

Let the spectroid $\mathcal{A}$ be given by the quiver from Figure 1 equipped with the relation $\gamma \beta$. If we make inductive one-point extensions along the sequence $(1,2,3,4)$, then Theorem 5 always applies and therefore $\mathcal{A}$ is completely preprojective. If we use the sequence $(1,3,4)$, then we obtain a completely preprojective initial subspectroid $\mathcal{B}$, but now the simple projective radical $P_{1}$ of $P_{2}$ has a nonsectional path to $P_{4}$ in the finite preprojective Auslander-Reiten quiver $\Gamma_{\mathcal{B}}$, which we display in Figure 2 As usual, the Auslander-Reiten translation is indicated by dotted arrows.

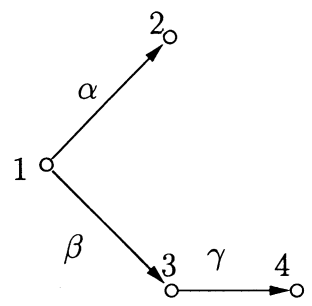

FiguRE 1.

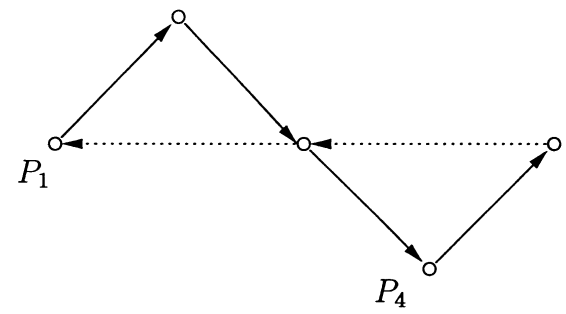

FIGURE 2.

4.2. We inductively define an ascending sequence $\mathcal{A}_{0}, \mathcal{A}_{1}, \ldots$ of initial subspectroids of $\mathcal{A}$, starting with the empty spectroid as $\mathcal{A}_{0}$, which we agree to be completely preprojective. For $n>0$ we distinguish two cases.

Case 1: $\mathcal{A}_{n-1}$ is not completely preprojective. If this happens, we put $\mathcal{A}_{n}:=$ $\mathcal{A}_{n-1}$. 
Case 2: $\mathcal{A}_{n-1}$ is completely preprojective. We define a quiver $Q_{n}$ with three types of vertices. The first type of vertices consists of all $x$ in $\mathcal{A}$ but not in $\mathcal{A}_{n-1}$ but $\operatorname{rad} P_{x}$ is a $\mathcal{A}_{n-1}$-module. The second type of vertices is given by all $x$ in $\mathcal{A}$ but not in $\mathcal{A}_{n-1}$ such that there is an indecomposable direct summand $U$ of $\operatorname{rad} P_{x}$ which belongs to a preprojective component of $\Gamma_{\mathcal{A}_{n-1}}$. There is only one vertex of the third type, which is a vertex $\infty$ different from all objects of $\mathcal{A}$. Note that there may be vertices which are of the first and second type simultaneously.

This set of vertices is equipped with four types of arrows. First, for all $x, y$ in $\mathcal{A}$ we have an arrow $\sigma_{x, y}: x \rightarrow y$ provided that $y$ is a proper successor of $x$ in $Q_{\mathcal{A}}$. Second, for all $x, y$ in $\mathcal{A}$ there is an arrow $\sigma_{x, y}^{\prime}: x \rightarrow y$ if there is a preprojective component of $\Gamma_{\mathcal{A}_{n-1}}$ containing an indecomposable direct summand $U$ of $\operatorname{rad} P_{x}$ and an indecomposable direct summand $V$ of $\operatorname{rad} P_{y}$ such that there is a nonsectional path from $U$ to $V$. Third, for all $x$ in $\mathcal{A}$ such that $\operatorname{rad} P_{x}$ is an $\mathcal{A}_{n-1}$-module there is an arrow $\sigma_{\infty, x}: \infty \rightarrow x$ provided that there is an indecomposable direct summand of $\operatorname{rad} P_{x}$ which does not lie in a preprojective component of $\Gamma_{\mathcal{A}_{n-1}}$. Fourth, there is a loop $\sigma_{\infty, \infty}: \infty \rightarrow \infty$.

We continue, again distinguishing two subcases.

Subcase 2.1: $Q_{n}$ does not have sources. We put $\mathcal{A}_{n}:=\mathcal{A}_{n-1}$.

Subcase 2.2: $Q_{n}$ has sources. We choose a source $x$ and define $\mathcal{A}_{n}$ to be the full subspectroid of $\mathcal{A}$ given by all objects $z$ in $\mathcal{A}_{n-1}$ and $x$.

Finally, we define $\mathcal{A}^{\star}$ to be the union of all the subcategories $\mathcal{A}_{n}$, and state some easy observations.

Remark. (a) The sequence $\mathcal{A}_{0} \subseteq \mathcal{A}_{1} \subseteq \cdots$ is a strictly ascending sequence of full subspectroids of $\mathcal{A}$.

(b) There is a nonnegative integer $n^{\star}$ which is at most the number of vertices of $Q_{\mathcal{A}}$ such that $\mathcal{A}^{\star}=\mathcal{A}_{n^{\star}}$.

(c) Each source of $Q_{\mathcal{A}}$ lies in $\mathcal{A}^{\star}$.

4.3. Before analysing the subspectroids $\mathcal{A}_{n}$, we want to establish a technical lemma. To formulate it, we suppose that $\mathcal{A}$ is a finite spectroid with cycle-free quiver and $\mathcal{B}$ is an initial subspectroid. By $\mathcal{C}$ we denote the full subspectroid of $\mathcal{A}$ given by all objects $x$ not belonging to $\mathcal{B}$. The subspectroid $\mathcal{C}$ is final in the obvious sense. The $\mathcal{C}$ - $\mathcal{B}$-bimodule $B$ is obtained by restricting the $\mathcal{A}$ - $\mathcal{A}$-bimodule $\mathcal{A}(-,-)$ to $\mathcal{B}$ in the first and to $\mathcal{C}$ in the second argument. We recall that modules in mod $-\mathcal{A}$ can be interpreted as triples $U=\left(U_{\omega}, \gamma_{U}, U_{\alpha}\right)$, where $U_{\omega}$ is in $\bmod -\mathcal{C}$, $U_{\alpha}$ in $\bmod -\mathcal{B}$ and $\gamma_{U}$ in $\operatorname{Hom}_{\mathcal{C}}\left(U_{\omega}, \operatorname{Hom}_{\mathcal{B}}\left(B, U_{\alpha}\right)\right)$. More precisely, an equivalence from $\bmod -\mathcal{A}$ to the category of these triples is given by sending an $\mathcal{A}$-module $U$ to its restriction $U_{\omega}$ to $\mathcal{C}$, its restriction $U_{\alpha}$ to $\mathcal{B}$ and the morphism $\gamma_{U}$ which maps for $x$ in $\mathcal{C}$ an element $u$ in $U_{\omega}(x)$ to $\mathcal{A}(y, x) \rightarrow U_{\alpha}(y), a \mapsto U(a)(u)$. Note that $\operatorname{Hom}_{\mathcal{B}}\left(B, U_{\alpha}\right)$ is considered as an object of $\bmod -\mathcal{C}$ using the $\mathcal{C}$-module structure of $B$ in the usual way.

We need the following easy observation. If $U=\left(U_{\omega}, \gamma_{U}, U_{\alpha}\right)$ is an indecomposable module in $\bmod -\mathcal{A}$ with $U_{\omega} \neq 0$, then for each indecomposable summand $U^{\prime}$ of $U_{\alpha}$ there is an object $x$ of $\mathcal{C}$ such that $\operatorname{Hom}_{\mathcal{B}}\left(\operatorname{res}_{\mathcal{B}} P_{x}, U^{\prime}\right) \neq 0$. The reason is that otherwise the triple $\left(0,0, U^{\prime}\right)$ would be a nontrivial direct summand of $U$.

Lemma 6. If $x$ is in $\mathcal{C}$ and $U$ is an indecomposable direct summand of $\operatorname{rad} P_{x}$ satisfying $U \neq \operatorname{res}_{\mathcal{B}} U$, then for each indecomposable direct summand $U^{\prime}$ of $\operatorname{res}_{\mathcal{B}} U$ there exists an object $y$ of $\mathcal{C}$ which is a proper predecessor of $x$ in $Q_{\mathcal{A}}$ and such that there is an indecomposable summand $V$ of $\operatorname{rad} P_{y}$ satisfying $\operatorname{Hom}_{\mathcal{B}}\left(\operatorname{res}_{\mathcal{B}} V, U^{\prime}\right) \neq 0$. 
In particular, for each $x$ in $\mathcal{C}$ and each indecomposable direct summand $U^{\prime}$ of $\operatorname{res}_{\mathcal{B}} P_{x}$ there is an object $y$ of $\mathcal{C}$ which is a predecessor of $x$ in $Q_{\mathcal{A}}$ and such that there is an indecomposable direct summand $V^{\prime}$ of $\operatorname{rad} P_{y}$ which is a $\mathcal{B}$-module and a predecessor of $U^{\prime}$ in ind $-\mathcal{B}$.

Proof. We define $\mathcal{A}^{\prime}$ as the full subspectroid of $\mathcal{A}$ given by all proper predecessors of $x$ and $\mathcal{B}^{\prime}$ as the full subspectroid of $\mathcal{A}^{\prime}$ given by all objects from $\mathcal{B}$. We observe that $\mathcal{A}^{\prime}$ and $\mathcal{B}^{\prime}$ are initial subspectroids of $\mathcal{A}$. Moreover, $\operatorname{rad} P_{x}$ and therefore also $U$ are actually $\mathcal{A}^{\prime}$-modules. Applying the remarks above to the indecomposable summand $U^{\prime}$ of $\operatorname{res}_{\mathcal{B}^{\prime}} U=U_{\alpha}$, we obtain an object $y$ in $\mathcal{A}^{\prime}$ but not in $\mathcal{B}^{\prime}$ satisfying $\operatorname{Hom}_{\mathcal{B}^{\prime}}\left(\operatorname{res}_{\mathcal{B}^{\prime}} P_{y}, U^{\prime}\right) \neq 0$. Since $\operatorname{res}_{\mathcal{B}^{\prime}} P_{y}=\operatorname{res}_{\mathcal{B}} P_{y}$ for all $y$ in $\mathcal{A}^{\prime}$, we also get $\operatorname{Hom}_{\mathcal{B}}\left(\operatorname{res}_{\mathcal{B}} P_{y}, U^{\prime}\right) \neq 0$. Because $y$ does not belong to $\mathcal{B}$, we know that res $P_{\mathcal{B}} P_{y}=$ $\operatorname{res}_{\mathcal{B}} \operatorname{rad} P_{y}$, which shows the existence of an indecomposable summand $V$ of $\operatorname{rad} P_{y}$ with $\operatorname{Hom}_{\mathcal{B}}\left(\operatorname{res}_{\mathcal{B}} V, U^{\prime}\right) \neq 0$.

In order to prove the additional assertion, we define $l(x)$ to be the number of proper predecessors of $x$ in $Q_{\mathcal{A}}$ which do not belong to $\mathcal{B}$. If $l(x)=0$, then $\operatorname{rad} P_{x}=$ $\operatorname{res}_{\mathcal{B}} P_{x}$, and the claim is clear using $y=x$. Now we suppose $l(x)>0$ and consider an indecomposable direct summand $U^{\prime}$ of $\operatorname{res}_{\mathcal{B}} P_{x}$. We find an indecomposable direct summand $U$ of $\operatorname{rad} P_{x}$ such that $U^{\prime}$ is a summand of $\operatorname{res}_{\mathcal{B}} U$.

If $\operatorname{res}_{\mathcal{B}} U=U$, then $U=U^{\prime}$ and we choose $y=x$ again. Otherwise we apply the lemma to obtain a proper predecessor $y$ of $x$ in $Q_{\mathcal{A}}$ lying in $\mathcal{C}$, such that there is an indecomposable direct summand $V$ of $\operatorname{rad} P_{y}$ satisfying $\operatorname{Hom}_{\mathcal{B}}\left(\operatorname{res}_{\mathcal{B}} V, U^{\prime}\right) \neq 0$. Hence there is also an indecomposable summand $V^{\prime}$ of $\operatorname{res}_{\mathcal{B}} V$ with $\operatorname{Hom}_{\mathcal{B}}\left(V^{\prime}, U^{\prime}\right) \neq$ 0 . The module $V^{\prime}$ is an indecomposable direct summand of $\operatorname{res}_{\mathcal{B}} P_{y}$ where $l(y)<$ $l(x)$. By induction there is a predecessor $y^{\prime}$ of $y$ in $Q_{\mathcal{A}}$ not belonging to $\mathcal{B}$ such that there is an indecomposable summand $V^{\prime \prime}$ of $\operatorname{rad} P_{y^{\prime}}$ which is a predecessor of $V^{\prime}$ in ind $-\mathcal{B}$.

4.4. After this preparation we are ready to study the subspectroids $\mathcal{A}_{n}$.

Lemma 7. For every integer $n \geq 0$ the following assertions hold:

(1) $\mathcal{A}_{n}$ is an initial subspectroid of $\mathcal{A}$.

(2) $\mathcal{A}_{n}$ is completely preprojective.

(3) If $n>0$ and $\mathcal{A}_{n}$ is a one-point extension of $\mathcal{A}_{n-1}$ by $x$, then the full subquiver of $\Gamma_{\mathcal{A}_{n}}$ whose vertex set consists of the predecessors of $P_{x}$ is a predecessor closed full subquiver of $\Gamma_{\mathcal{A}}$ such that $\tau_{\mathcal{A}} X=\tau_{\mathcal{A}_{n}} X$ for all its vertices $X$.

Proof. We apply induction on $n$ and observe that for $n=0$ nothing needs to be proved. For $n>0$ we know by induction that (1), (2), (3) hold for $\mathcal{A}_{n-1}$. Thus we are in case 2 of the algorithm. For subcase $2.1, \mathcal{A}_{n}=\mathcal{A}_{n-1}$, we are done. Thus there is a source $x$ of $Q_{n}$ such that the the objects of $\mathcal{A}_{n}$ are obtained from the objects of $\mathcal{A}_{n-1}$ by adding $x$. We decompose $P_{x}=\bigoplus_{i=1}^{n} U_{i}$ into indecomposable direct summands $U_{i}$.

On (1): For each object $z$ of $\mathcal{A}$ which does not belong to $\mathcal{A}_{n-1}$ such that $\operatorname{rad} P_{z}$ is not in $\bmod -\mathcal{A}_{n-1}$, there exists a proper predecessor $y$ in $Q_{\mathcal{A}}$ not belonging to $\mathcal{A}_{n-1}$. Therefore there is an arrow $\sigma_{y, z}$ in $Q_{n}$, showing that $z$ is not a source. Consequently, $\operatorname{rad} P_{x}$ is a $\mathcal{A}_{n-1}$-module and all proper predecessors of $x$ in $Q_{\mathcal{A}}$ are in $\mathcal{A}_{n-1}$, yielding that $\mathcal{A}_{n}$ is initial as well.

On (2): We want to apply Theorem [5, and have to check the conditions (a), (b), (c). 
The absence of an arrow $\infty \rightarrow x$ shows that all summands $U_{i}$ lie in preprojective components, which is (a). The failure of (b) would mean that there is a nonsectional path from a summand $U_{i}$ to a summand $U_{j}$ yielding a loop in $x$. For (c) we assume the existence of a nonsectional path from some $U_{j}$ to $P_{z}$ where $z$ is in $\mathcal{A}_{n-1}$. Then there is also an indecomposable summand $V$ of $\operatorname{rad} P_{z}$ such that there is a nonsectional path from $U_{j}$ to $V$. By the inductive construction of $\mathcal{A}_{n-1}$ there has to be $t<n$ such that $\mathcal{A}_{t}$ is the one-point extension of $\mathcal{A}_{t-1}$ by $z$. By induction we know that property (3) of the lemma holds for $\mathcal{A}_{t}$. Hence, there is a nonsectional path from $U_{j}$ to $V$ in the preprojective components of the Auslander-Reiten quiver of $\mathcal{A}_{t-1}$. In particular, $U_{j}$ is an $\mathcal{A}_{t-1}$-module, and we obtain an arrow $x \rightarrow z$ in $Q_{t}$. This is a contradiction to $z$ being chosen as source of $Q_{t}$.

On (3): It suffices to show that each right minimal and right almost split morphism $g: Y \rightarrow X$ in $\bmod -\mathcal{A}_{n}$, where $X$ occurs as predecessor of $P_{x}$ in $\Gamma_{\mathcal{A}_{n}}$, is actually right minimal and right almost split in $\bmod -\mathcal{A}$. For $X$ projective this is obvious. For $X$ not projective it is enough to prove that $\tau_{\mathcal{A}} X=\tau_{\mathcal{A}_{n}} X$. If we assume that this equality does not hold, then the formula $\tau_{\mathcal{A}} X=R \tau_{\mathcal{A}_{n}} X$ shows the existence of an object $y$ in $\mathcal{A}$ but not in $\mathcal{A}_{n}$ satisfying $\operatorname{Hom}_{\mathcal{A}_{n}}\left(\operatorname{res}_{\mathcal{A}_{n}} P_{y}, \tau_{\mathcal{A}_{n}} X\right) \neq 0$. We obtain the existence of an indecomposable direct summand $U$ of $\operatorname{rad} P_{y}$ and an indecomposable direct summand $U^{\prime}$ of the restriction $\operatorname{res}_{\mathcal{A}_{n}} U$ such that there is a nonsectional path from $U^{\prime}$ to some $U_{i}$. By Lemma 6 there is another object $z$ not belonging to $\mathcal{A}_{n}$ which has an indecomposable summand $V^{\prime}$ of $\operatorname{rad} P_{z}$ which itself is an $\mathcal{A}_{n}$-module and a predecessor of $U^{\prime}$ in ind $-\mathcal{A}_{n}$. Altogether we found a nonsectional path from $V^{\prime}$ to $U_{i}$ in the preprojective component of $\mathcal{A}_{n-1}$. Thus $z$ is a predecessor of $x$ in $Q_{n}$, which contradicts the fact that $x$ is a source.

Remark. Part (2) of the lemma shows that in the algorithm case 1 never occurs, and part (3) implies that the predecessors of $P_{x}$ in ind $-\mathcal{A}$ are precisely the predecessors of $P_{x}$ in the preprojective components of $\Gamma_{\mathcal{A}_{n}}$.

Since $\mathcal{A}^{\star}=\mathcal{A}_{n^{\star}}$, the lemma immediately yields our first main result.

Theorem 8. Suppose $\mathcal{A}$ is a finite spectroid with cycle-free quiver. Then the following assertions hold:

(1) $\mathcal{A}^{\star}$ is an initial subspectroid of $\mathcal{A}$.

(2) $\mathcal{A}^{\star}$ is completely preprojective.

(3) For all $x$ in $\mathcal{A}^{\star}$ the predecessors of $P_{x}$ in the preprojective components of $\Gamma_{\mathcal{A}^{\star}}$ are precisely the predecessors of $P_{x}$ in ind $-\mathcal{A}$.

\section{Properties of $\mathcal{A}^{\star}$}

5.1. We continue to study finite spectroids $\mathcal{A}$ whose quivers are cycle-free. In the previous section we introduced an algorithm producing an initial subspectroid $\mathcal{A}^{\star}$ which is completely preprojective. The construction of $\mathcal{A}^{\star}$ depends on the choices of sources in the quivers $Q_{n}$. Consequently, it seems possible that for different choices of sources the algorithm yields different subspectroids $\mathcal{A}^{\star}$. Our first aim in this section is to show that this is not the case.

We define $X$ in ind $-\mathcal{A}$ to be predecessor bounded if there is a bound for the length $n$ of paths $X_{0} \rightarrow X_{1} \rightarrow \cdots \rightarrow X_{n}=X$ in ind $-\mathcal{A}$ ending in $X$. Otherwise $X$ is called predecessor unbounded. Clearly, $X$ is predecessor bounded if it lies in a preprojective component.

The following proposition follows immediately from the work of Liu (see $\underline{\mathrm{Li}}$ ). 
Proposition 9. Let $\mathcal{A}$ be a finite spectroid. If $\Omega$ is a component of $\Gamma_{\mathcal{A}}$ not containing any projective vertex, then each module $X$ in $\Omega$ is predecessor unbounded.

Proof. The component $\Omega$ is semiregular in the notation of [Li]. If $\Omega$ does not contain an oriented cycle, then it is even a left stable component with this property, and by [Li theorem 3.3] for each vertex $X$ of $\Omega$ all the modules $\tau_{\mathcal{A}}^{m} X$ with $m \geq 0$ are pairwise different. In particular, $X$ is predecessor unbounded.

In the case that $\Omega$ contains an oriented cycle, by $[\mathrm{Li}$, theorem 3.6] it is a stable or coextended tube as introduced in Ri]. It is easy to see that each object $X$ of $\Omega$ is predecessor unbounded.

Let us return to our algorithm.

Corollary 10. If $\sigma_{\infty, x}$ is an arrow in $Q_{n}$ with $x \neq \infty$, then $P_{x}$ is predecessor unbounded in ind $-\mathcal{A}$.

Proof. The existence of the arrow $\sigma_{\infty, x}$ means that there is a radical summand $U_{i}$ of $P_{x}$ which does not belong to any preprojective component of $\mathcal{A}_{n-1}$. On the other hand, these preprojective components contain all indecomposable projective $\mathcal{A}_{n-1}$-modules. Therefore the component containing $U_{i}$ satisfies the assumptions of proposition 9 Thus $U_{i}$ is predecessor unbounded in ind $-\mathcal{A}_{n-1}$. Since $P_{x}$ is a successor of $U_{i}$ in ind $-\mathcal{A}$ and $\mathcal{A}_{n-1}$ is an initial subspectroid of $\mathcal{A}$, we obtain that $P_{x}$ is predecessor unbounded in ind $-\mathcal{A}$.

5.2. In an analogous fashion as for ind $-\mathcal{A}$, we say that a vertex $x$ of a quiver $Q$ is predecessor bounded provided there is a bound for the length $n$ of paths $x_{0} \rightarrow$ $x_{1} \rightarrow \cdots \rightarrow x_{n}=x$ in $Q$ ending in $x$.

Lemma 11. If $x \neq \infty$ is a predecessor unbounded vertex of $Q_{n}$, then $P_{x}$ is predecessor unbounded in ind $-\mathcal{A}$.

Proof. We start out by proving the following claim: If $\alpha: x \rightarrow y$ is an arrow in $Q_{n}$ with $x, y \neq \infty$, then $P_{x}$ is a predecessor of $P_{y}$ in ind $-\mathcal{A}$.

The claim is obvious, if $\alpha=\sigma_{x, y}$ comes from a path in $Q_{\mathcal{A}}$. Otherwise $\alpha=\sigma_{x, y}^{\prime}$ such that there is a nonsectional path $U=Z_{0} \rightarrow Z_{1} \rightarrow \cdots \rightarrow Z_{m}=V$ from an indecomposable direct summand $U$ of $\operatorname{rad} P_{x}$ to an indecomposable direct summand $V$ of $\operatorname{rad} P_{y}$ in a preprojective component of $\mathcal{A}_{n-1}$. We may choose such a nonsectional path and an index $t$ with $Z_{t}=\tau_{\mathcal{A}_{n-1}} Z_{t+2}$ having the property that there is no nonsectional path from $U$ to $Z_{t}$. By [HR, proposition 2] we get $\operatorname{Hom}_{\mathcal{A}_{n-1}}\left(U, Z_{t}\right) \neq 0$ and thus $0 \neq \operatorname{Hom}_{\mathcal{A}_{n-1}}\left(\operatorname{res}_{\mathcal{A}_{n-1}} P_{x}, Z_{t}\right)=R Z_{t}(x)=\tau_{\mathcal{A}} Z_{t+2}(x)$. The Yoneda lemma yields a path from $P_{x}$ to $P_{y}$ in ind $-\mathcal{A}$.

Now we proceed with the proof of the lemma. That $x$ is predecessor unbounded shows that for each integer $m \geq 0$ there is a path $x_{0} \rightarrow x_{1} \rightarrow \cdots \rightarrow x_{m}=x$ in $Q_{n}$. If for some $m$ the vertex $\infty$ appears as $x_{t}$ and without loss of generality we assume that $x_{l} \neq \infty$ for all $l>t$, then by corollary 10 and the claim $P_{x}$ is predecessor unbounded in ind $-\mathcal{A}$. In the case that all the paths can be formed without using $\infty$, because of the finiteness of $\mathcal{A}$ we find a path such that there exists $t>0$ with $x_{0}=x_{t}$. Using the claim, this gives rise to a cycle of indecomposable projective modules starting and ending in $P_{x_{0}}$. Again by the claim, this makes $P_{x}$ predecessor unbounded in ind $-\mathcal{A}$. 
Theorem 12. Let $\mathcal{A}$ be a finite spectroid with a cycle-free quiver. The set of objects of $\mathcal{A}^{\star}$ consists precisely of those objects of $\mathcal{A}$ such that $P_{x}$ is predecessor bounded.

Proof. Since $\mathcal{A}^{\star}$ is completely preprojective, $P_{x}$ is obviously predecessor bounded in ind $-\mathcal{A}^{\star}$ for each $x$ in $\mathcal{A}^{\star}$. Part (3) of Theorem 8 shows that this is also true in ind $-\mathcal{A}$. Conversely, if $x$ is not in $\mathcal{A}^{\star}=\mathcal{A}_{n^{\star}}$, then by possibly passing to an appropriate predecessor in $Q_{\mathcal{A}}$ we may suppose that $x$ belongs to $Q_{n^{\star}}$. The assumption that $x$ is predecessor bounded implies that the full subquiver of $Q_{n^{\star}}$ given by the predecessors of $x$ is cycle-free and consequently has a source which is a source of $Q_{n^{\star}}$ as well, a contradiction. Thus $x$ is a predecessor unbounded vertex of $Q_{n^{\star}}$ and therefore $P_{x}$ is predecessor unbounded in ind $-\mathcal{A}$ by Lemma 11].

Corollary 13. $\mathcal{A}$ is completely preprojective if and only if $\mathcal{A}=\mathcal{A}^{\star}$.

The characterisation of $\mathcal{A}^{\star}$ given in the theorem could be used to define $\mathcal{A}^{\star}$ abstractly. One is tempted to say that $\mathcal{A}^{\star}$ is something like a maximal initial completely preprojective subspectroid of $\mathcal{A}$. As a warning against this misconception and an illustration of the algorithm, we present an example showing that there may exist initial completely preprojective subspectroids which properly contain $\mathcal{A}^{\star}$. Let $\mathcal{A}$ be given by the quiver from Figure 3 subjected to the relations $\beta \alpha, \beta^{\prime} \alpha^{\prime}$, and $\delta \varphi$.

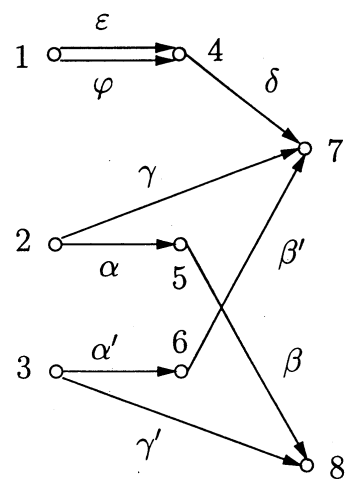

FIGURE 3 .

It turns out that $\mathcal{A}^{\star}=\mathcal{A}_{6}$ is the full subspectroid supported by $\{1,2,3,4,5,6\}$. The quiver $Q_{7}$ is shown in Figure 4. The one-point extension of $\mathcal{A}^{\star}$ by 8 is still completely preprojective and an initial subspectroid of $\mathcal{A}$.

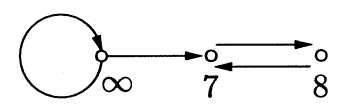

FIGURE 4. 
5.3. The following theorem summarises our findings.

Theorem 14. Let $\mathcal{A}$ be a finite spectroid with cycle-free quiver.

(a) If $\Theta$ is a preprojective component of $\Gamma_{\mathcal{A}^{\star}}$ such that for each $x$ in $\mathcal{A} \backslash \mathcal{A}^{\star}$ no indecomposable direct summand of $\operatorname{rad} P_{x}$ belongs to $\Theta$, then $\Theta$ is a preprojective component of $\Gamma_{\mathcal{A}}$.

(b) If $\Theta$ is a preprojective component of $\Gamma_{\mathcal{A}}$, then $\Theta$ is a preprojective component of $\Gamma_{\mathcal{A}^{*}}$ such that for each $x$ in $\mathcal{A} \backslash \mathcal{A}^{\star}$ no indecomposable direct summand of $\operatorname{rad} P_{x}$ belongs to $\Theta$.

Proof. By Lemma [6] we know that $\Theta$ does not contain any indecomposable direct summand of $\operatorname{res}_{\mathcal{A}^{\star}} P_{x}$ for all $x$ not in $\mathcal{A}^{\star}$. Thus (a) follows from Lemma 2 .

In order to see (b) we observe that all projectives $P_{z}$ in $\Theta$ are predecessor bounded. Hence the initial subspectroid induced by all $z$ such that $P_{z}$ belongs to $\Theta$ is contained in $\mathcal{A}^{\star}$. Consequently, $\Theta$ is a preprojective component of $\Gamma_{\mathcal{A}^{\star}}$. Assume there exists an indecomposable summand $U$ of $\operatorname{rad} P_{x}$ with $x$ not in $\mathcal{A}^{\star}$ such that $U$ appears in $\Theta$. Because $R U \neq U$, the module $U$ cannot be an injective $\mathcal{A}^{\star}$-module and therefore there is some nonprojective module $V$ in $\Theta$ such that $U=\tau_{\mathcal{A}^{\star}} V$. But this implies $\tau_{\mathcal{A}} V=R \tau_{\mathcal{A}^{\star}} V \neq \tau_{\mathcal{A}^{\star}} V$, which is a contradiction.

5.4. A finite spectroid $\mathcal{A}$ of global dimension at most 1 is called hereditary. By Ga1 it is of the form $\mathcal{A}=k Q$, where $Q$ is a finite cycle-free quiver. It is well-known (see e.g. [Ri]) that hereditary spectroids are completely preprojective. We want to use our setting to reprove this classical result.

An enumeration $\left\{x_{1}, \ldots, x_{r}\right\}$ of the set of objects of a completely preprojective spectroid $\mathcal{A}$ is called a construction if $j \geq i$ provided that $x_{i}$ is a predecessor of $x_{j}$ in $Q_{\mathcal{A}}$. Equivalently, this means that $\mathcal{A}$ can be built up by one-point extensions along this enumeration. Our algorithm from Section 4 produces special constructions which we call strong constructions. The example in subsection 4.1 shows that in general not all constructions are strong.

Theorem 15. If $\mathcal{A}$ is a hereditary finite spectroid, then every construction of $\mathcal{A}$ is strong. In particular, $\mathcal{A}$ is completely preprojective.

Proof. We want to show that, given a construction $\left\{x_{1}, \ldots, x_{r}\right\}$ for all $n=0, \ldots, r$, the subspectroid $\mathcal{A}_{n}$ may be chosen as the full subspectroid of $\mathcal{A}$ induced by $\left\{x_{1}, \ldots, x_{n}\right\}$. For this purpose by induction it is sufficient to prove that $x_{n}$ is always a source of $Q_{n}$. The heredity of $\mathcal{A}$ shows that all summands of $\operatorname{rad} P_{x_{n}}$ are projective and predecessors of projective modules are projective. Therefore arrows of type $\sigma_{\infty, x}$ and $\sigma_{x, y}^{\prime}$ for $x, y \neq \infty$ do not exist.

Remark. Our algorithm will also work for the much more general class of Artin algebras (see $\underline{\mathrm{ARS}}$ ) if one is able to calculate the dimension vectors of the indecomposable projective modules and the indecomposable direct summands of their radicals.

\section{REFERENCES}

[AR] M. Auslander, I. Reiten, Representation theory of Artin algebras III, Commun. Algebra 3 (1975), 239-294. MR 52:504

[ARS] M. Auslander, I. Reiten, S.O. Smalø, Representation theory of Artin algebras, Cambridge, 1995. MR 96c:16015

[BL] R. Bautista, F. Larrión, Auslander-Reiten quivers for certain algebras of finite representation type, J. London Math. Soc. 26 (1982), 43-52. MR 83k:16014 
[BGRS] R. Bautista, P. Gabriel, A.V. Roiter, L. Salmerón, Representation-finite algebras and multiplicative bases, Invent. Math. 81 (1985), 217-285. MR 87g:16031

[Bo] K. Bongartz, A criterion for finite representation type, Math Ann. 269 (1984), 1-12. MR 86k:16023

[BG] K. Bongartz, P. Gabriel, Covering spaces in representation theory, Invent. Math. 65 (1982), 331-378. MR 84i:16030

[DR] V. Dlab, C.M. Ringel, Indecomposable representations of graphs and algebras, Mem. Amer. Math. Soc. 173 (1973). MR 56:5657

[DP] P. Dräxler, J.A. de la Peña, One the existence of postprojective components in the Auslander-Reiten quiver of an algebra, Tsukuba J. Math. 20 (2) (1996), 457-469. MR 98a:16020

[Ga1] P. Gabriel, Unzerlegbare Darstellungen I, Manuscr. Math. 6 (1972), 71-103. MR 48:11212

[Ga2] P. Gabriel, Auslander-Reiten sequences and representation-finite algebras, Lecture Notes in Math. 831 (1980), 1-71. MR 82i:16030

[GR] P. Gabriel, A.V. Roiter, Representations of finite-dimensional algebras, Encyclopedia of the Mathematical Sciences, Vol. 73, Algebra VIII, A.I. Kostrikin and I.V. Shafarevich (Eds.), Berlin, Heidelberg, New York, 1992, pp. 1-177. MR 94h:16001b

[Ha] D. Happel, Composition factors for indecomposable modules, Proc. Amer. Math. Soc. 86 (1982), 29-31. MR 84i:16031

[HR] D. Happel, C.M. Ringel, Directing projective modules, Arch. Math. 60 (1993), 237-246. MR 94b:16016

[KP] S. Kasjan, J.A. de la Peña, Constructing the preprojective components of an algebra, J. Algebra 179 (1996), 793-807. MR 97c:16015

[Li] S. Liu, Shapes of connected components of the Auslander-Reiten quivers of Artin algebras, Representation theory of algebras and related topics (Mexico City, 1994), 109-137, CMS Conf. Proc., 19, Amer. Math. Soc., Providence, RI, 1996. MR 97e:16037

[Ri] C.M. Ringel, Tame algebras and integral quadratic forms, Springer LNM 1099 (1984). MR 87f:16027

[Sc] T. Scheuer, The canonical decomposition of the poset of a hammock, J. London Math. Soc. (2) 49 (1994), 232-243. MR 95a:16017

Fakultät für Mathematik, Universität Bielefeld, P.O. Box 100131, D-33501 BieleFELD, GERMANY

Fakultät für Mathematik, Universität Bielefeld, P.O. Box 100131, D-33501 BieleFeld, Germany 